\title{
Prognostic relevance of MOG antibodies in children with an acquired demyelinating syndrome
}

\section{(1) $\mathbb{6}$}

Eva-Maria Hennes, MD

Matthias Baumann, MD

Kathrin Schanda, MSc

Banu Anlar, MD

Barbara Bajer-Kornek, MD

Astrid Blaschek, MD

Sigrid Brantner-Inthaler, MD

Katharina Diepold, MD

Astrid Eisenkölbl, MD

Thaddäus Gotwald, MD

Georgi Kuchukhidze, MD

Ursula Gruber-Sedlmayr, MD

Martin Häusler, MD

Romana Höftberger, MD

Michael Karenfort, MD

Andrea Klein, MD

Johannes Koch, MD

Verena Kraus, MD

Christian Lechner, MD

Steffen Leiz, MD

Frank Leypoldt, MD, $\mathrm{PhD}$

Simone Mader, PhD

Klaus Marquard, MD

Imke Poggenburg, MD

Daniela Pohl, MD, PhD

Martin Pritsch, MD

Markus Raucherzauner, $\mathrm{MD}$

Mareike Schimmel, MD

Charlotte Thiels, MD

Daniel Tibussek, MD

Silvia Vieker, MD

Carolin Zeches, MD

Thomas Berger, MD

Markus Reindl, PhD

Kevin Rostásy, MD

On behalf of the

BIOMARKER

Study Group

\section{ABSTRACT}

Objective: To assess the prognostic value of MOG antibodies (abs) in the differential diagnosis of acquired demyelinating syndromes (ADS).

Methods: Clinical course, MRI, MOG-abs, AQP4-abs, and CSF cells and oligoclonal bands (OCB) in children with ADS and 24 months of follow-up were reviewed in this observational prospective multicenter hospital-based study.

Results: Two hundred ten children with ADS were included and diagnosed with acute disseminated encephalomyelitis (ADEM) $(n=60)$, neuromyelitis optica spectrum disorder (NMOSD) $(n=12)$, clinically isolated syndrome (CIS) $(n=101)$, and multiple sclerosis (MS) ( $n=37)$ after the first episode. MOG-abs were predominantly found in ADEM (57\%) and less frequently in NMOSD (25\%), CIS (25\%), or MS (8\%). Increased MOG-ab titers were associated with younger age $(p=0.0001)$, diagnosis of ADEM ( $p=0.005)$, increased CSF cell counts $(p=0.011)$, and negative OCB ( $p=0.012)$. At 24-month follow-up, 96 children had no further relapses. Thirtyfive children developed recurrent non-MS episodes (63\% MOG-, 17\% AQP4-abs at onset). Seventy-nine children developed MS (4\% MOG-abs at onset). Recurrent non-MS episodes were associated with high MOG-ab titers $(p=0.0003)$ and older age at onset $(p=0.024)$. MS was predicted by MS-like MRI $(p<0.0001)$ and OCB $(p=0.007)$. An MOG-ab cutoff titer $\geq 1: 1,280$ predicted a non-MS course with a sensitivity of $47 \%$ and a specificity of $100 \%$ and a recurrent non-MS course with a sensitivity of $46 \%$ and a specificity of $86 \%$.

Conclusions: Our results show that the presence of MOG-abs strongly depends on the age at disease onset and that high MOG-ab titers were associated with a recurrent non-MS disease course. Neurology ${ }^{\circledR}$ 2017;89:900-908

\section{GLOSSARY}

abs = antibodies; ADEM = acute disseminated encephalomyelitis; ADEMON = acute disseminated encephalomyelitis with optic neuritis; $\mathbf{A D S}$ = acquired demyelinating syndromes; CIS = clinically isolated syndrome; EDSS = expanded disability score; FLAIR = fluid-attenuated inversion recovery; LETM = longitudinal extensive transverse myelitis; MDEM = multiphasic acute disseminated encephalomyelitis; MOG = myelin oligodendrocyte glycoprotein; $\mathbf{M S}$ = multiple sclerosis; $\mathbf{N M O S D ~ = ~}$ neuromyelitis optica spectrum disorder; OCB = oligoclonal bands; $\mathbf{O N}=$ optic neuritis; OND = other neurologic diseases; $\mathbf{T M}=$ transverse myelitis.

The differential diagnosis of children with acquired demyelinating syndromes (ADS) encompasses monophasic diseases such as acute disseminated encephalomyelitis (ADEM), clinically isolated syndromes (CIS), or potentially relapsing forms such as multiple sclerosis (MS). Early diagnosis is important because of other disorders mimicking a first episode of ADS and different treatment options. In this context, antibodies (abs) to myelin oligodendrocyte glycoprotein (MOG) have regained attention. Serum MOG-abs can be detected in children with monophasic ADEM with MOG-abs that drop to undetectable levels. ${ }^{1-3}$ MOG-abs have further been detected in aquaporin (AQP)-4-ab-seronegative NMOSD, recurrent optic neuritis $(\mathrm{ON})$, transverse myelitis $(\mathrm{TM}),{ }^{4-7}$ and multiphasic acute disseminated encephalomyelitis (MDEM), but less frequently in adults with these disorders. ${ }^{2,8-10}$ Two recent studies revealed that MOG-abs are nearly exclusively detectable in children with monophasic or relapsing events other than MS. ${ }^{11,12}$

Author affiliations are provided at the end of the article.

Coinvestigators are listed at Neurology.org.

Go to Neurology.org for full disclosures. Funding information and disclosures deemed relevant by the authors, if any, are provided at the end of the article. 
Correspondence to Dr. Rostásy:

k.rostasy@kinderklinik-datteln.de

Supplemental data at Neurology.org
In the current study, we investigated the prevalence and predictive value of MOG-abs in children with a first ADS in combination with clinical and MRI features at onset and outcome after 2 years.

METHODS Between 2009 and 2014, serum samples of 302 children with a suspected ADS were sent to us from 60 different medical centers in Germany, Austria, Italy, Switzerland, Turkey, and Canada for testing of MOG- and AQP4-abs and included in an ongoing prospective study. Two hundred ten children with a data set including a diagnosis of ADS, clinical presentation, CSF studies (cell count, oligoclonal bands [OCB]), cerebral MRI from the first episode, MOG- and AQP4-ab status up to 3 months after first episode, treatment history, final diagnosis, and clinical outcome with an expanded disability score (EDSS) after at least 24 months were selected. Clinical data at onset and follow-up were obtained in the majority of cases with a standardized questionnaire or a medical discharge summary from the referring physician.

Ninety-two children were excluded for the following reasons: (1) 2 children with ADEM died at disease onset; (2) 14 children were diagnosed with other neurologic diseases (OND) (e.g., limbic encephalitis); (3) 11 children presented as having ADS but were later diagnosed with OND; (4) in 23 patients, the initial serum sample was not taken during the first 3 months; (5) in 2 children, necessary data from disease onset were not available; (6) 40 children were lost to follow-up (figure 1). Based on the initial clinical information, radiologic evaluation and AQP4-ab status children were assigned to one of the following diagnoses based on the International Paediatric MS Study Group (IPMSSG) recommendations ${ }^{13}$ : (1) 60 patients with ADEM; (2) 101 patients with CIS divided further into 6 subcategories (isolated $\mathrm{ON}, \mathrm{n}=42$; bilateral $\mathrm{ON}, \mathrm{n}=6$; transverse myelitis, $\mathrm{n}$ $=20$; brainstem, $\mathrm{n}=7$; cerebral, $\mathrm{n}=11$; polysymptomatic, $\mathrm{n}=$ 15); (3) 37 patients were diagnosed with MS because of radiologic evidence of dissemination in space and time ${ }^{14}$; (4) 12 patients with NMOSD ${ }^{15}$ (figure 1 and table e-1 at Neurology.org). MRI studies were assessed by at least 2 reviewers unaware of the clinical presentation and MOG-ab status (M.B., T.G., and G.K.) including the following sequences: cranial MRI of all patients in T2-, fluid-attenuated inversion recovery (FLAIR)-axial, T2-sagittal, T1-axial with contrast-medium sequences in addition to spinal T2- and T1-sagittal with contrast-medium sequences available from 160/210 children. Clinical follow-up including EDSS, presence of cognitive dysfunction (e.g., attentional problems), or epilepsy was obtained through medical records or a standardized questionnaire at least after 24 months. Demographic and clinical findings of 63/210 children from this cohort were reported already in 4 studies focusing on the presence of MOG-abs in $\operatorname{NMOSD}\left(\mathrm{n}=2^{7}\right.$ and $\left.\mathrm{n}=30^{16}\right)$, ADEM $\left(\mathrm{n}=28^{1}\right)$, and MDEM $\left(\mathrm{n}=3^{10}\right)$. We decided to include these children because of new clinical data (e.g., further relapses and additional serum samples).

Standard protocol approvals, registrations, and patient consents. The study was approved by the Ethics Committee of the Medical University of Innsbruck, Austria (study number AN4059). All caregivers provided written informed consent.

Antibody assays. Serum samples were analyzed for the presence of MOG- and AQP4-abs by live cell-based immunofluorescence assays as described previously. ${ }^{8}$ MOG-abs were tested using fulllength MOG ( $\alpha-1$ isoform) and IgG (heavy and light chains, Dianova)-specific secondary abs. Screening was performed at dilutions of 1:20 and 1:40 by at least 2 independent clinically blinded investigators (K.S., S.M., and M.R.), and positive serum samples were further diluted in 2-fold increments to determine the end-point titers. Titer levels of $\geq 1: 160$ were classified as MOG-ab positive as described previously. ${ }^{8}$ Using heavy chainspecific secondary abs for IgM, IgG (Dianova), and IgG1 (Invitrogen), we excluded an isolated IgM reactivity in borderline (1:160 and 1:320) seropositive samples. Two hundred eighteen follow-up samples from 126/210 patients taken at least 6 months after the initial event were analyzed for MOG-abs.

Statistical analysis. Statistical analysis was performed using IBM SPSS, release 22.0 (IBM Corporation). Primary outcome was the final classification after 24 months into 3 categories: monophasic, recurrent non-MS demyelinating episodes, or MS. The association of MOG-ab titers with clinical and immunologic parameters at onset was analyzed by ordinary regression analysis using the enter model with all parameters entered at the first step. The prognostic relevance of biomarkers, clinical or demographic parameters for the final classification after 24 months (monophasic, recurrent non-MS, or MS), was tested by multinominal logistic regression analysis with all parameters entered at the first step. Furthermore, the association of demographic, clinical, and immunologic parameters with the disease course after 24 months was analyzed using univariate statistical tests $\left(\chi^{2}\right.$ test, Fisher exact test, Kruskal-Wallis test, 1-way analysis of variance, and Wilcoxon signed-rank test).

RESULTS Patients. Children with ADS and a complete data set were included (figure 1). The cohort of 210 children consisted of 120 female and 90 male patients with a median age at onset of 12.0 years (range 0.5-17). Sixty patients were diagnosed with ADEM, 101 with CIS, and 12 with NMOSD. Thirty-seven patients with CIS had radiologic evidence of dissemination in space and time and were thus diagnosed with MS (figure 1 and table e-1). After 24 months, the final diagnosis remained ADEM in $46 / 60$ children. Fourteen of 60 children had further events and were diagnosed with $\operatorname{NMOSD}(\mathrm{n}=1)$, MS $(n=2), \operatorname{MDEM}(n=8)$, or acute disseminated encephalomyelitis with optic neuritis (ADEMON) $(n=3)$. Fifty-four of 101 children with CIS had further demyelinating episodes: 11 children with $\mathrm{ON}$ had further episodes of $\mathrm{ON}$ and were diagnosed with recurrent $\mathrm{ON}$. Three children with $\mathrm{ON}$ or longitudinal extensive transverse myelitis (LETM) had additional episodes of ON or LETM, increasing the number of children with NMOSD to 16 after 2 years. Forty children with CIS were diagnosed with MS due to clinical relapses $(\mathrm{n}=35)$ or new FLAIR/T2 lesions on follow-up MRI ( $\mathrm{n}=5)$ (figure 1).

Comparison of clinical and immunologic features according to MOG-ab status at baseline. Serum MOG-abs with a titer $\geq 1: 160$ were detected in 65/210 (31\%) patients with a median titer of 1:1,280 (range 1:160 1:40,960). Patients with high MOG-ab titers had a lower age at onset (Spearman $\rho=-0.416, p<$ 0.0001). Accordingly, MOG-ab titers were highest in 


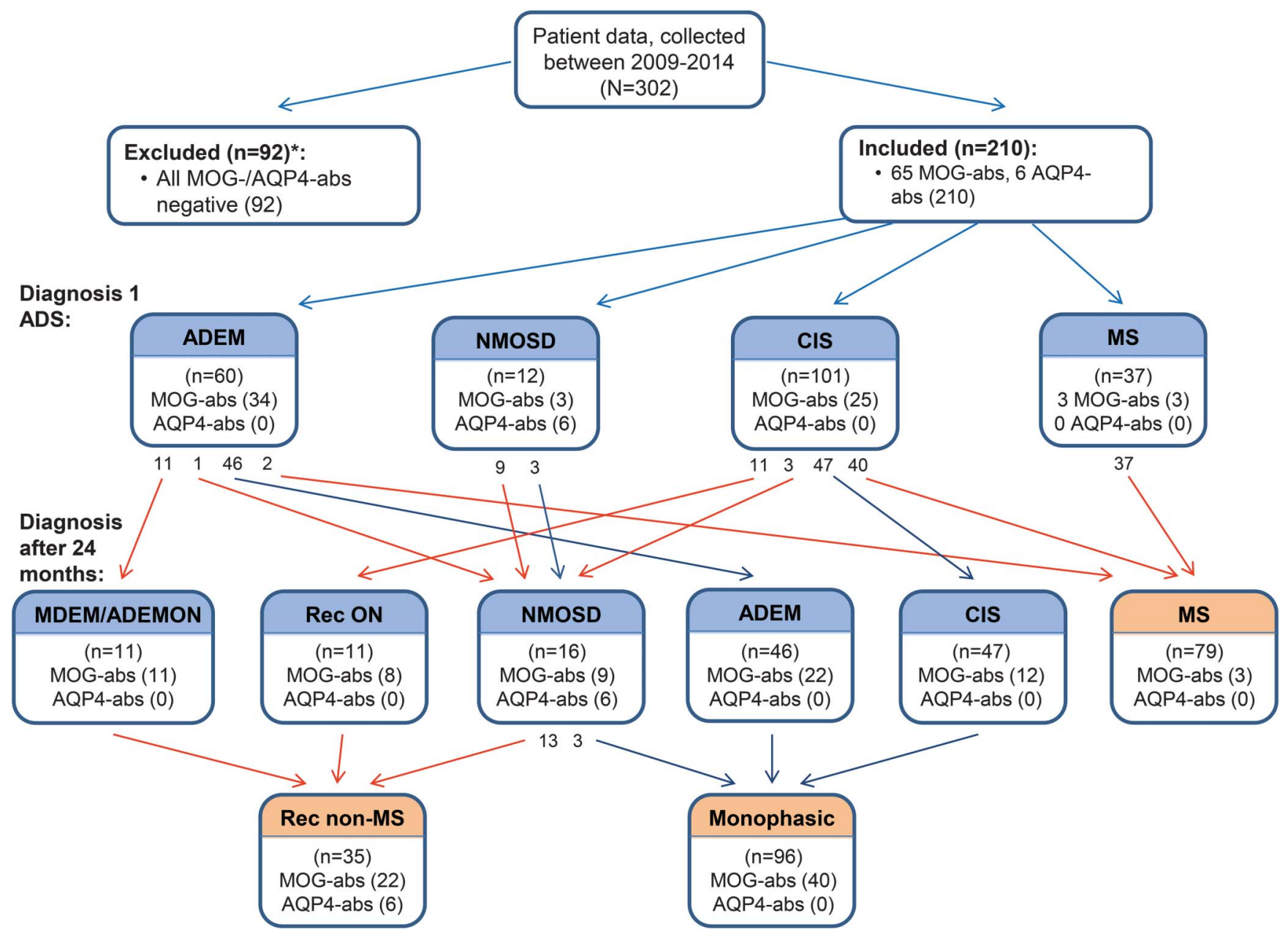

Initial diagnoses of ADEM, NMOSD, CIS, and MS changed during follow-up of at least 24 months to monophasic diseases (n = 96), recurrent non-MS diseases $(n=35)$, or MS ( $n=79$ ). Patients with a recurrent non-MS disease course more often were seropositive for MOG-abs $(n=22 / 35)$ compared with a monophasic disease course $(n=40 / 96)$ or MS $(n=3 / 79)$. *92/302 patients were excluded because of the following reasons: (1) death at first episode $(n=2)$; (2) other inflammatory neurologic disease at onset $(n=14)$; (3) ADS at onset, final diagnosis OND ( $n=11)$; (4) no serum sample within first 3 months ( $n=23)$; (5) incomplete data set $(n=2)$; and (6) lost to follow-up $(n=40)$. Blue arrows: monophasic disease course; red arrows: recurrent disease course. abs = antibodies; $\mathrm{ADEM}=$ acute disseminated encephalomyelitis; $\mathrm{ADEMON}=$ acute disseminated encephalomyelitis with optic neuritis; $\mathrm{ADS}=$ acute demyelinating syndrome; AQP4 = aquaporin-4; CIS = clinically isolated syndrome; Dx = diagnoses; MDEM = multiphasic disseminated encephalomyelitis; MOG = myelin oligodendrocyte glycoprotein; $\mathrm{MS}=$ multiple sclerosis; $\mathrm{n}=$ number; NMOSD = neuromyelitis optica spectrum disorder; ON = optic neuritis; $\mathrm{OND}=$ other neurologic disease; $r$ rec $=$ recurrent.

children younger than 5 years and lower in older children (figure 2).

MOG-ab titers were highest in children with ADEM (figure 2). Because previous studies have analyzed smaller patient cohorts using explorative statistical methods, we decided to analyze the association of MOG-ab titer levels $(0-80,160-640$, and $\geq 1: 1,280)$ using a multivariate model (table 1). High MOG-ab titers were associated with younger age ( $p=0.00001)$, diagnosis of $\operatorname{ADEM}(p=0.005)$, absence of OCB $(p=$ $0.012)$, and higher CSF cell counts $(p=0.011)$. By contrast, diagnosis of CIS with MS-like MRI or MS was associated with MOG-ab seronegativity or low titers. MOG-ab-positive children with CIS and MSatypical MRI exclusively presented with $\mathrm{ON}(\mathrm{n}=18)$ or TM $(\mathrm{n}=7)($ table 1$)$.
Prognostic parameters for a final diagnosis of MS or other forms of recurrent demyelinating syndromes after 24 months. Next, we studied the prognostic value of baseline markers for final disease classification in the entire MOG-ab-positive and MOG-ab-negative cohort (table 2 and table e-2). After 24 months, 96 children still had a monophasic diseases course $(42 \%$ MOG-abs at onset), 35 children developed further demyelinating episodes other than MS (63\% MOGabs and 17\% AQP4-abs at onset), and 79 children were diagnosed with MS (4\% MOG-abs at onset).

Multinominal logistic regression analysis indicated that a recurrent non-MS course was predicted by higher MOG-ab titers $(p=0.0003)$ and older age at onset $(p=0.024)$ compared with the monophasic group (table 2). By contrast, MS was predicted by an 
Figure 2 Association of MOG-ab titers with age at onset (A), assigned diagnosis (B), and clinical course at follow-up (C, D)
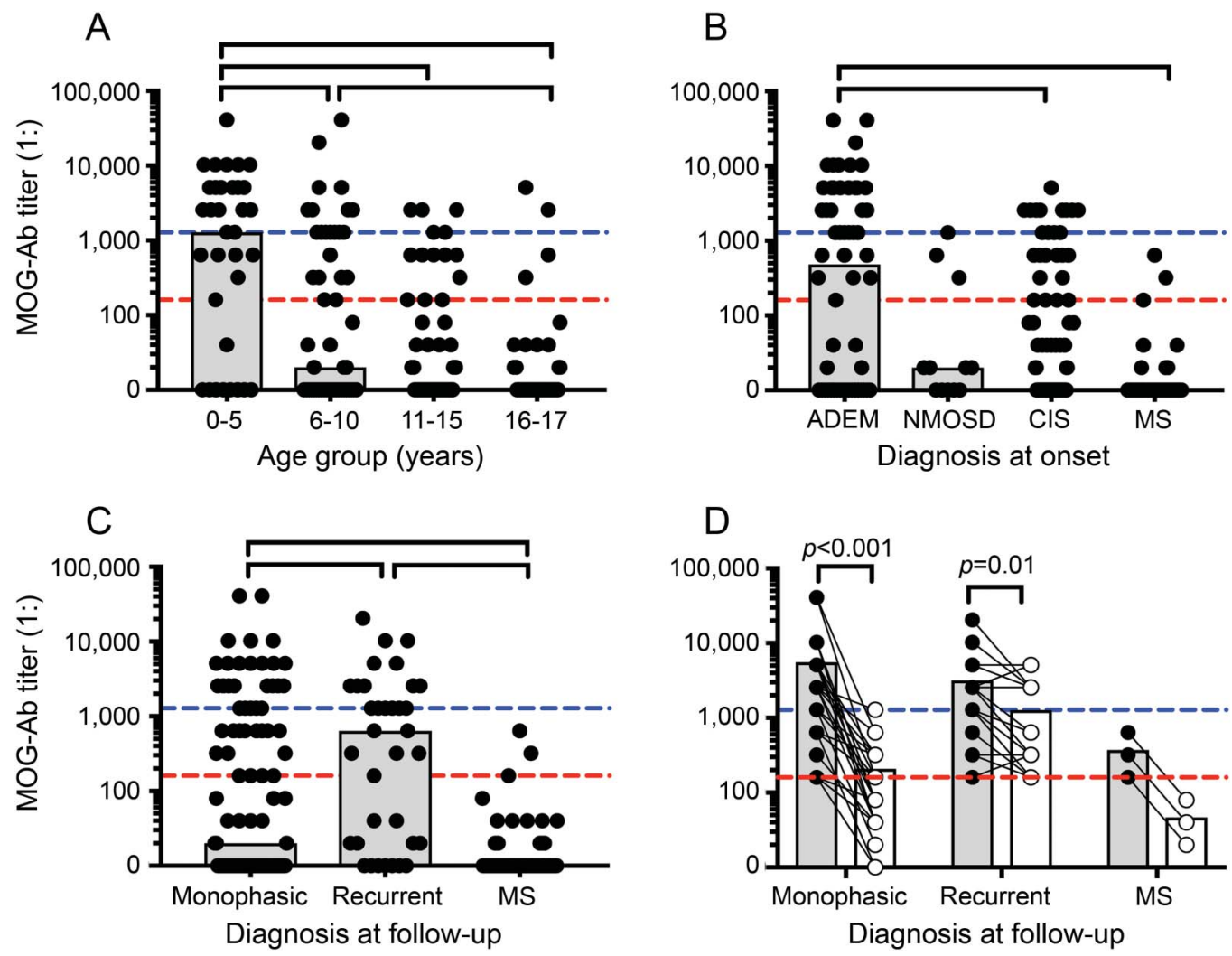

ADEM = acute disseminated encephalomyelitis; $\mathrm{CIS}=$ clinically isolated syndrome; MOG = myelin oligodendrocyte glycoprotein; MS = multiple sclerosis; NMOSD = neuromyelitis optica spectrum disorder.

MS-like MRI $\left(p=4 \times 10^{-9}\right)$ and OCB at onset $(p=$ 0.007) compared with the monophasic group with no significant role of MOG-abs. Of note, 28/35 (80\%) patients with a recurrent non-MS course had abs to either MOG (63\%) or AQP4 (17\%), and 27/35 (77\%) patients were females (table 2). Thus, patients with a higher age (median 10 years), female sex, MOG- or AQP4-abs, and MS-atypical MRI at onset have a high risk of developing a non-MS-like recurrent disease course independent from the initial diagnosis. Furthermore, these patients had a higher risk for ON during the course of disease $(26 / 35,74 \%)$ compared with patients with a monophasic disease course $(34 / 96,35 \%)$ or MS (19/79, 24\%). After 24 months, no significant difference in the EDSS or other clinical residuals was noted between the different groups (tables e-2 and e-4). Patients in the MOGab-negative recurrent non-MS group (most of them with NMOSD with AQP4-abs) and in the group with MOG-ab titers $\geq 1,280$ had the shortest time to relapse. Fourteen of 35 (40\%) patients in the recurrent non-MS group were treated with IV immunoglobulin $(\mathrm{n}=3)$, rituximab $(\mathrm{n}=3$, AQP4-positive NMOSD), or azathioprine $(\mathrm{n}=4)$ compared with $3 / 96(3 \%)$ in the monophasic group or $60 / 79(76 \%)$ in the MS group.
Finally, we analyzed whether different MOG-ab cutoff titer levels provided prognostic information on the disease course after 2 years. Although our currently used MOG-ab cutoff value of $\geq 1: 160$ had the highest sensitivity for predicting a non-MS course or a recurrent non-MS course after 2 years, the corresponding specificities were modest (table 3 ). By contrast, a cutoff value of $\geq 1: 1,280$ had a lower sensitivity for predicting a non-MS course or a recurrent non-MS course, but the corresponding specificities were much better.

Disease course of MOG-ab-seropositive patients over 24-month follow-up. Forty of 65 (61\%) MOG-abseropositive children had a monophasic disease course. Twenty-two children (34\%) developed a recurrent nonMS disease course and only 3 children (5\%) were diagnosed with MS (table e-3). MOG-ab-positive patients with a recurrent non-MS disease course were diagnosed with MDEM or ADEM-ON ( $\mathrm{n}=11)$, followed by recurrent ON $(\mathrm{n}=8)$ and $\operatorname{NMOSD}(\mathrm{n}=$ 3). Sixteen of $22(73 \%)$ patients with a recurrent disease course had $\mathrm{ON}$ at beginning or during follow-up compared with $13 / 40(32 \%)$ patients with a monophasic disease and 2/3 (66\%) with MS. Twenty-one of 22 MOG-ab-positive patients with a recurrent non-MS 


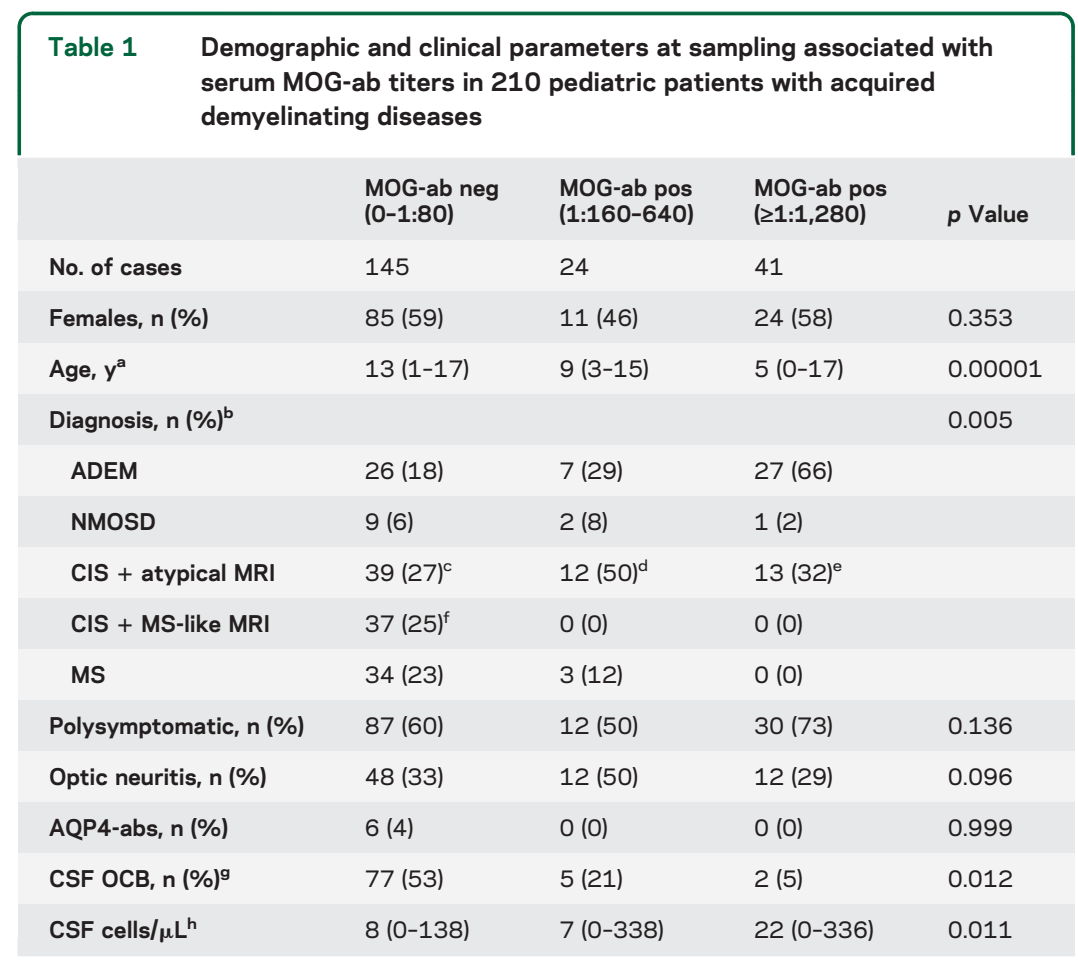

Abbreviations: $a b=$ antibody; $\mathrm{ADEM}=$ acute disseminated encephalomyelitis; $\mathrm{AQP}-4=$ aquaporin-4; $\mathrm{CIS}$ = clinically isolated syndrome; MOG = myelin oligodendrocyte glycoprotein; $\mathrm{MS}$ = multiple sclerosis; neg = seronegative; NMOSD = neuromyelitis optica spectrum disorder; $\mathrm{OCB}=$ oligoclonal lgG bands; $\mathrm{ON}=$ optic neuritis; pos $=$ seropositive; $\mathrm{TM}=$ trans verse myelitis.

Groups were statistically compared using ordinal regression analysis with all parameters entered at the first step (enter method). $\mathrm{R}^{2}=0.378$ (Cox and Snell), 0.468 (Nagelkerke), 0.289 (McFadden); model $\chi^{2}=99.73 ; p=5 \times 10^{-18}$.

${ }^{a}$ Median (range).

${ }^{\mathrm{b}}$ Diagnosis at onset and MRI at onset were analyzed together because they were highly correlated, and MRI was used to establish the diagnosis of MS.

c Symptoms: brainstem (1), ON (24), polysymptomatic (3), and TM (11).

d Symptoms: ON (8) and TM (4).

e Symptoms: ON (10) and TM (3).

f Symptoms: brainstem (6), cerebral (11), ON (6), polysymptomatic (12), and TM (2).

${ }^{9}$ Six missing cases were included in the analysis with values entered as samples means.

${ }^{\mathrm{h}}$ Sixteen missing cases were included in the analysis with values entered as samples means (data shown as median with range).

disease course had a clinical relapse after 6 months (1-13 months) and 2.2 relapses (1-5 relapses) in the first 2 years, which was not different compared with other recurrent groups (table e-3). Of note, an MS-like MRI (e.g., lesions perpendicular to the long axis of the corpus callosum) was absent in the monophasic and recurrent non-MS patients. ${ }^{14}$ MOG-ab titers at presentation were similar in MOG-ab-positive children with a monophasic vs a recurrent non-MS disease course and lower in patients with MS.

Correlation of MOG-ab follow-up titers with the clinical disease course. Finally, we analyzed 218 follow-up serum samples from 75/145 (52\%) MOG-abnegative patients and 51/65 (78\%) MOG-abpositive patients (cutoff $\geq 1: 160$ ). MOG-ab-negative patients did not develop MOG-abs over time. Overall, 36/51 (71\%) MOG-ab-positive patients still had MOG-abs at final follow-up with an overall decline of the median titer from 1:1,280 (range 16040,960 ) at onset to $1: 160$ (range $0-5,120$ ). MOGabs titers dropped from onset to follow-up in all groups, with the strongest effect seen in the monophasic and MS groups, but remained highest in the recurrent non-MS group (figure 2). When using a cutoff value of $\geq 1: 1,280$ as calculated above, 33/ 126 (26\%) patients were MOG-ab positive at onset, but only 7/126 (6\%) still had MOG-abs at follow-up. Nineteen of 48 monophasic patients had MOG-abs $\geq 1: 1,280$ at onset, but only 1 patient (2\%) had persisting abs, whereas $15 / 26$ patients with a recurrent non-MS course had MOG-abs at onset (58\%), 6 of them (23\%) with persisting abs. None of the 52 patients with MS had MOG-abs $\geq 1: 1,280$ at onset or follow-up.

DISCUSSION We report the frequency of serum MOG-abs in a cohort of 210 children presenting with ADS prospectively followed for at least 2 years. We confirm previous findings such as the presence of MOG-abs in one-third of all children with ADS and that MOG-abs are associated with a non-MS-like disease course. ${ }^{11,12}$ As in previous studies, we observed a strong correlation between age and MOG-ab seropositivity at disease onset, ${ }^{3,8,11,12,17,18}$ revealing that, in particular, children younger than 10 years with an acute non-MS-like episode harbor MOG-abs (table e-4). Furthermore, we show that a subgroup of children continued to have further demyelinating episodes such as recurrent ON, MDEM, ADEMON, or NMOSD characterized by the presence of MOG-abs and less often AQP4-abs, higher age at onset, and female predominance. Recurrent episodes in most cases were accompanied by $\mathrm{ON}$, occurred in the context of persisting, high-titer MOG-abs, and in the first year after the initial event. The characteristics of this subgroup of patients are similar to those recently reported in adult MOG-ab-positive patients. ${ }^{19,20}$ To evaluate what characterizes children with MOG-abs who develop a relapsing non-MS-like disease course, we compared them with children with a monophasic disease course. The initial features of both groups had notable differences: children with monophasic disease course were younger, more often males, and less often had involvement of optic nerves. At initial presentation, antibody titers in children with transient MOG-abs were similar to those with persistent MOG-abs but declined in the following months. Taking all aspects into account, 2 subgroups with MOG-abs emerge, as already suggested in a smaller cohort of pediatric patients ${ }^{17}$ : One subgroup characterized by children with a median age at onset of 4.5 years and equal sex distribution is primarily diagnosed with ADEM and less often with CIS associated with a monophasic course and transient MOG-abs; the 


\begin{tabular}{|c|c|c|c|}
\hline \multirow[t]{2}{*}{$\begin{array}{l}\text { Predictive factc } \\
210 \text { pediatric } p\end{array}$} & \multicolumn{3}{|c|}{$\begin{array}{l}\text { Predictive factors at disease onset for a recurrent non-MS disease course or MS after } 24 \text { months in } \\
210 \text { pediatric patients with acquired demyelinating diseases }\end{array}$} \\
\hline & Monophasic & Recurrent non-MS & MS \\
\hline No. of cases, $n(\%)$ & $96(45.7)$ & 35 (16.7) & 79 (37.6) \\
\hline Females, n (\%) & 47 (49); reference & 27 (77); $p=0.102$ & $46(58) ; p=0.551$ \\
\hline Age at onset, $y^{a}$ & 7 (0.5-17); reference & $\begin{array}{l}10(2-17) ; p=0.024 \\
O R=1.17(1.02,1.34)\end{array}$ & $14(1-17) ; p=0.070$ \\
\hline \multicolumn{4}{|l|}{ MOG-abs at onset, n (\%) } \\
\hline Negative (0-1:80), n (\%) & $56(58)$ & $13(37)$ & $76(96)$ \\
\hline Positive (1:160-640), n (\%) & $15(16)$ & $6(17)$ & $3(4)$ \\
\hline Positive $(\geq 1: 1,280)$ & 25 (26); reference & $\begin{array}{l}16(46) ; p=0.0003 \\
O R=4.02(1.90,8.49)\end{array}$ & $0(0) ; p=0.194$ \\
\hline AQP4-abs at onset, n (\%) & $\mathrm{O}(0)$; reference & $6(17) ; p=0.993$ & $0(0) ; p=0.999$ \\
\hline CSF OCB at onset, $n(\%)^{b}$ & 15 (16); reference & $3(9) ; p=0.396$ & $\begin{array}{l}66(84) ; p=0.007 \\
O R=6.69(1.70,26.34)\end{array}$ \\
\hline CSF cells/ $/ \mu$ at onset ${ }^{c}$ & $10(0-338)$ & $17(0-232) ; p=0.187$ & $12(0-70) ; p=0.982$ \\
\hline MS-typical MRI at onset, $n$ (\%) & 4 (4); reference & $0(0) ; p=0.975$ & $\begin{array}{l}71(90) ; p=4 \times 10^{-9} \\
O R=63.04(15.86,250.52)\end{array}$ \\
\hline Polysymptomatic onset, n (\%) & 62 (65); reference & $20(57) ; p=0.448$ & $47(60) ; p=0.686$ \\
\hline Optic neuritis at onset, $\mathrm{n}(\%)$ & 34 (35); reference & $20(57) ; p=0.203$ & $18(23) ; p=0.126$ \\
\hline
\end{tabular}

Abbreviations: abs = antibodies; $\mathrm{ADEM}=$ acute disseminated encephalomyelitis; $\mathrm{AQP}-4$ = aquaporin-4; $\mathrm{CIS}=$ clinically isolated syndrome; MOG = myelin oligodendrocyte glycoprotein; $\mathrm{MS}=$ multiple sclerosis; NMOSD = neuromyelitis optica spectrum disorder; OCB = lgG bands; $\mathrm{ON}=$ optic neuritis; $\mathrm{OR}=$ odds ratio with $95 \% \mathrm{Cl}$; $\mathrm{TM}=$ transverse myelitis. Groups were statistically compared using multinominal logistic regression analysis with all parameters entered at the first step (enter method). $\mathrm{R}^{2}=0.705$ (Cox and Snell), 0.809 (Nagelkerke), 0.596 (McFadden); model $\chi^{2}=256.28 ; p=4 \times 10^{-44}$. Diagnosis at baseline: monophasic $=46$ ADEM, 3 NMOSD, and 47 CIS (28 ON, 15 TM, 1 cerebral, and 3 polysymptomatic) patients; recurrent non-MS = 12 ADEM, 9 NMOSD, and $14 \mathrm{CIS}$ (13 ON and 1 TM); MS = 2 ADEM, 40 CIS (7 ON, 4 TM, 10 cerebral, 12 polysymptomatic, and 7 brainstem), and $37 \mathrm{MS}$.

Diagnosis at final follow-up: monophasic $=46$ ADEM, 3 NMOSD, and 47 CIS patients; recurrent non-MS = 13 NMOSD, 11 recurrent $\mathrm{ON}$, and $11 \mathrm{MDEM} / \mathrm{ADEM}-\mathrm{ON}$ patients.

${ }^{a}$ Median (range).

${ }^{b}$ Six missing cases were included in the analysis with values entered as samples means.

${ }^{\mathrm{c}}$ Sixteen missing cases were included in the analysis with values entered as samples means (data shown as median with range).

second subgroup consists of children who are older at onset (median 8 years) and predominantly females. $\mathrm{ON}$ at onset or later is common, and they are primarily diagnosed with ADEM or CIS but develop a relapsing non-MS course such as MDEM, ADEMON, NMOSD, or recurrent ON with persistent MOG-abs. Furthermore, our results suggest that patients with a recurrent non-MS disease course have higher MOG-ab titers at onset compared with patients with a monophasic course or MS and that persisting MOG-abs are predominantly present in pediatric patients with recurrent non-MS disease course. We conclude that persisting high-titer MOGabs are associated with a risk of relapse but not the sole presence at onset. Our results also suggest that a cutoff higher than previously anticipated, e.g., $\geq 1,280$, could be used to increase the specificity for a non-MS disease course. However, because this leads to a decrease in sensitivity improvement of MOG-ab testing, using more specific assays as recently published is needed. ${ }^{21}$ The presence of MOG-abs has also been described in adult patients with MS with a relapsing course involving the brainstem, spinal cord, and poor response to MS therapies. MOG-abs in this subgroup were present over a long time with fluctuation and reappearance of MOG-abs. The authors concluded that patients with MS with MOGabs represent a distinct phenotype benefiting from different treatment strategies. ${ }^{22}$ This is in line with biopsy findings in adults with fulminant episodes and MOG-abs revealing a lesion pattern reminiscent of MS type 2 with demyelination and presence of complement activation. ${ }^{23-25}$ Our observations are further supported by a recent study involving 50 adults with a history of $\mathrm{ON}$ and/or TM: Every fourth MOG-ab-positive patient with ON or myelitis had clinicoradiologic findings of MS, suggesting a phenotype overlap between patients with seropositive NMOSD and those with seropositive MS. ${ }^{19}$ Three children in our cohort had MS and MOG-abs (figure e-1). Two children with an MS-like MRI and positive OCB were diagnosed already at age 4 and 8 years. One had an isolated $\mathrm{ON}$ and the other patient a right-sided hemiplegia. The third patient was 14 


\begin{tabular}{|c|c|c|c|c|}
\hline \multirow[t]{3}{*}{$\begin{array}{l}\text { Prognostic } \\
\text { recurrent } r\end{array}$} & $\begin{array}{l}\text { nificance of MOG-ab } \\
\text { MS, and MS) }\end{array}$ & iter levels on the dise & ase course after 24 & lonths (monophasic, \\
\hline & \multicolumn{4}{|c|}{ MOG-ab titer level at baseline-cutoff values } \\
\hline & $\geq 1: 160$ & $\geq 1: 320$ & $\geq 1: 640$ & $\geq 1: 1,280$ \\
\hline Number, n (\%) & 65 (31) & $59(28)$ & $52(25)$ & $41(19)$ \\
\hline Age, $y^{a}$ & $6(0-17)$ & $6(0-17)$ & $5(0-17)$ & $5(0-17)$ \\
\hline \multicolumn{5}{|l|}{ Diagnosis at onset, $\mathrm{n}(\%)$} \\
\hline ADEM (60) & $34(57)$ & $33(55)$ & $30(50)$ & $27(45)$ \\
\hline NMOSD (12) & $3(25)$ & $3(25)$ & $2(17)$ & 1 (8) \\
\hline CIS (101) & $25(25)$ & $21(21)$ & $19(19)$ & $13(13)$ \\
\hline MS (37) & $3(8)$ & 2 (5) & 1 (3) & $0(0)$ \\
\hline \multicolumn{5}{|l|}{$\begin{array}{l}\text { Disease course (monophasic, } \\
\text { recurrent non-MS, MS) after } \\
2 \text { y, n (\%) }\end{array}$} \\
\hline Monophasic (96) & $40(42)$ & $36(37)$ & 33 (34) & $25(26)$ \\
\hline ADEM (46) & $22(48)$ & $21(46)$ & $20(43)$ & 18 (39) \\
\hline CIS (47) & 15 (32) & $12(25)$ & $11(23)$ & $6(13)$ \\
\hline NMOSD (3) & $3(100)$ & $3(100)$ & $2(67)$ & 1 (33) \\
\hline Recurrent non-MS (35) & $22(63)$ & $21(60)$ & $18(51)$ & $16(46)$ \\
\hline MDEM/ADEM-ON (11) & $11(100)$ & $11(100)$ & 9 (82) & $8(73)$ \\
\hline NMOSD (13) & $3(23)$ & $3(23)$ & $3(23)$ & $3(23)$ \\
\hline Recurrent ON (11) & $8(73)$ & $7(64)$ & $6(54)$ & $5(45)$ \\
\hline MS (79) & $3(4)$ & 2 (2) & 1 (1) & $0(0)$ \\
\hline \multicolumn{5}{|l|}{$\begin{array}{l}\text { Prediction of a non-MS } \\
\text { disease course (monophasic } \\
\text { or recurrent) after } 2 y\end{array}$} \\
\hline Sensitivity $(95 \% \mathrm{Cl})$ & $0.473(0.390,0.558)$ & $0.435(0.353,0.521)$ & $0.389(0.310,0.475)$ & $0.313(0.240,0.397)$ \\
\hline Specificity $(95 \% \mathrm{Cl})$ & $0.962(0.894,0.990)$ & $0.975(0.912,0.996)$ & $0.987(0.932,0.999)$ & $1.0(0.954,1.0)$ \\
\hline$p^{b}$ & $<0.0001$ & $<0.0001$ & $<0.0001$ & $<0.0001$ \\
\hline \multicolumn{5}{|l|}{$\begin{array}{l}\text { Prediction of a recurrent } \\
\text { non-MS disease course } \\
\text { after } 2 \text { y }\end{array}$} \\
\hline Sensitivity $(95 \% \mathrm{Cl})$ & $0.629(0.463,0.768)$ & $0.6(0.436,0.744)$ & $0.514(0.356,0.670)$ & $0.457(0.305,0.618)$ \\
\hline Specificity $(95 \% \mathrm{Cl})$ & $0.754(0.685,0.812)$ & $0.783(0.716,0.837)$ & $0.806(0.741,0.857)$ & $0.857(0.780,0.901)$ \\
\hline$p^{b}$ & $<0.0001$ & $<0.0001$ & 0.0002 & $<0.0001$ \\
\hline
\end{tabular}

Abbreviations: abs = antibodies; ADEM = acute disseminated encephalomyelitis; $A D E M O N=$ acute disseminated encephalomyelitis with optic neuritis; $\mathrm{CIS}$ = clinically isolated syndrome; MDEM = multiphasic disseminated encephalomyelitis; MOG = myelin oligodendrocyte glycoprotein; MS = multiple sclerosis; NMOSD = neuromyelitis optica spectrum disorder; OCB = lgG bands; ON = optic neuritis.

${ }^{\mathrm{a}}$ Median (range).

${ }^{\mathrm{b}}$ Calculated using the Fisher exact test.

years old with sensory disturbances in his legs due to TM. The 2 younger patients had frequent episodes not involving the brainstem or myelon. All patients are currently treated with MS therapies and MOGabs dropping to undetectable levels over time. Children with persistent MOG-abs in our study had more episodes with ON compared with children with absent or transient MOG-abs as previously reported.5,6,11,26,27 ON with MOG-abs has distinct features separating it from other diseases such as bilateral involvement, rapid visual impairment combined with good recovery after steroid treatment, and optic disc swelling. ${ }^{26,28-30}$ Several limitations need to be addressed: MRI studies were performed on scanners with different field strengths, potentially leading to different results. Serologic follow-up was not available from a large part of initially MOG-abnegative children, as well as from MOG-ab-positive patients without relapses. With a higher number of follow-up serum samples, further conclusions, i.e., regarding the correlation between disease activity and MOG-ab titers, would have been possible. We also 
have to mention that 63 children of our cohort were reported in previous studies, potentially leading to a selection bias. Nevertheless, the proportion of MOG-ab-positive children was similar to other reported cohorts. ${ }^{11,12}$

Since a previous publication indicated that MOGab reactivity in patients with MS might be caused by IgM abs detected by IgG heavy + light chain $(\mathrm{H}+$ L) reactive secondary abs, whereas IgG1 reactive secondary abs are more specific, ${ }^{21}$ we tested a subset of our samples with different secondary abs (table e-5). Although IgM abs were present in some of these samples, all were also IgG1 reactive. This finding is consistent with the work of Waters et al., ${ }^{21}$ who showed that almost all IgG1-reactive samples also have MOG-antibody titers $\geq 1: 160$ when measured using the $(\mathrm{H}+\mathrm{L})$ assay.

MOG-abs are present in a substantial number of very young children with $\mathrm{ADS}$ and are associated with a monophasic disease course in the context of declining antibody levels. High and persisting MOG-abs on the other hand are associated with a recurrent disease course other than MS. Collaborative studies are needed to better define this group of diseases with the common denominator MOG-abs.

\section{AUTHOR AFFILIATIONS}

From the Department of Pediatrics (E.-M.H., K.M.), Olga Hospital, Stuttgart, Germany; Division of Pediatric Neurology, Department of Pediatrics I (M.B., C.L.), Clinical Department of Neurology (K.S., G.K., T.B., M.R.), and Department of Neuroradiology (T.G.), Medical University of Innsbruck, Austria; Department of Pediatric Neurology (B. A.), Hacettepe University Faculty of Medicine, Ankara, Turkey; Institute of Neurology (B.B.-K., R.H.), Medical University of Vienna, Austria; Department of Pediatric Neurology and Developmental Medicine (A.B.), Dr. von Hauner's Children's Hospital, University of Munich, Germany; Department of Pediatrics (S.B.-I.), Donauspital, Vienna, Austria; Department of Pediatrics (K.D.), Division of Pediatric Neurology, Klinikum Kassel, Germany; Department of Pediatrics (A.E.), Women's and Children's Hospital, Linz, Austria; Department of Pediatrics (U.G.-S.), LKH Medical University Graz, Austria; Department of Pediatrics (M.H.), Division of Neuropediatrics and Social Pediatrics, Medical University RWTH Aachen, Germany; Department of General Pediatrics, Neonatology and Pediatric Cardiology (M.K.), University Children's Hospital, Heinrich-Heine-University, Düsseldorf, Germany; Department of Neuropediatrics (A.K.), University Children's Hospital Basel and Bern, Switzerland; Department of Pediatrics (J.K.), Salzburger Landeskliniken (SALK) and Paracelsus Medical University (PMU), Austria; Children's Hospital Schwabing (V.K.), Technische Universität München, Germany; Department of Pediatrics (S.L.), Division of Pediatric Neurology, Klinikum Dritter Orden, Munich, Germany; Neuroimmunology (F.L.), Institute of Clinical Chemistry and Department, Medical University Schleswig-Hostein, Kiel, Germany; Center for Autoimmune and Musculoskeletal Diseases (S.M.), The Feinstein Institute for Medical Research, Manhasset, NY; Department of Pediatric Neurology (I.P.), Elisabeth Children's Hospital, Medical University Oldenburg, Germany; Department of Neurology (D.P.), Children's Hospital of Eastern Ontario, University of Ottawa, Canada; Department of Neuropediatrics (M.P.), Children's Hospital DRK Siegen, Germany; Department of Pediatrics (M.R.), Kliniken OstallgäuKaufbeuren, Germany; Division of Pediatric Neurology (M.S.), Children's Hospital, Klinikum Augsburg, Germany; Department of Neuropediatrics (C.T.), University Children's Hospital, Ruhr-University Bochum, Germany; Department of Pediatrics (D.T.), Zentrum für Seltene Erkrankungen ZSE, Heinrich Heine University Düsseldorf, Germany; Department of Pediatrics
(S.V.), Division of Pediatric Neurology, Klinikum Bayreuth, Germany; Department of Pediatrics (C.Z.), Division of Pediatric Neurology, Schoen Klinikum Vogtareuth, Germany; and Department of Pediatric Neurology (E.-M.H., K.R.), Vestische Kinder- und Jugendklinik Datteln, University Witten/Herdecke, Germany.

\section{AUTHOR CONTRIBUTIONS}

Eva-Maria Hennes: acquisition of data, study supervision, and analysis and interpretation of data. Matthias Baumann: acquisition of data, analysis and interpretation of data, and critical revision of the manuscript for intellectual content. Kathrin Schanda: acquisition of data and analysis and interpretation of data. Banu Anlar, Barbara Bajer-Kornek, Astrid Blaschek, Sigrid Brantner-Inthaler, Katharina Diepold, Astrid Eisenkölbl, Thaddäus Gotwald, Georgi Kuchukhidze, Ursula Gruber-Sedlmayr, Martin Häusler, Romana Höftberger, Michael Karenfort, Andrea Klein, Johannes Koch, Verena Kraus, Christian Lechner, Steffen Leiz, Frank Leypoldt, Simone Mader, Klaus Marquard, Imke Poggenburg, Daniela Pohl, Martin Pritsch, Markus Raucherzauner, Mareike Schimmel, Charlotte Thiels, Daniel Tibussek, Silvia Vieker, and Carolin Zeches: acquisition of data and critical revision of the manuscript for intellectual content. Thomas Berger: acquisition of data, analysis and interpretation of data, and critical revision of the manuscript for intellectual content. Markus Reindl: acquisition of data, analysis and interpretation of data, and study supervision. Kevin Rostásy: study concept and design, acquisition of data, analysis and interpretation of data, and study supervision.

\section{STUDY FUNDING}

Supported by grant numbers 14158 and 15918 from the Jubilaeumsfonds of the Austrian National Bank (Kevin Rostasy) and research grant "BIG WIG MS" from the Austrian Federal Ministry of Science, Research and Economy (Markus Reindl).

\section{DISCLOSURE}

E. Hennes, M. Baumann, K. Schanda, B. Anlar, B. Bajer-Kornek, A. Blaschek, S. Brantner-Inthaler, K. Diepold, A. Eisenkölbl, T. Gotwald, G. Kuchukhidze, U. Gruber-Sedlmayr, M. Häusler, R. Höftberger, M. Karenfort, A. Klein, J. Koch, V. Kraus, C. Lechner, and S. Leiz report no disclosures relevant to the manuscript. F. Leypoldt reports speaker's honoraria from Grifols, Biogen, Bayer, Roche, and Teva, advisory board positions for Roche and Biogen. F. Leypoldt's institute performs commercial antibody testing without receiving any personal financial benefit. S. Mader, K. Marquard, I. Poggenburg, D. Pohl, M. Pritsch, M. Raucherzauner, M. Schimmel, C. Thiels, D. Tibussek, S. Vieker, and C. Zeches report no disclosures relevant to the manuscript. T. Berger: The Neurological Research Laboratory (Medical University of Innsbruck and Tirol Kliniken, Markus Reindl, and Thomas Berger) receives payments for antibody assays (AQP4- and anti-neuronal antibodies) and for AQP4- and MOG-antibody validation experiments organized by Euroimmun (Germany). M. Reindl: The Neurological Research Laboratory (Medical University of Innsbruck and Tirol Kliniken, Markus Reindl, and Thomas Berger) receives payments for antibody assays (AQP4- and anti-neuronal antibodies) and for AQP4- and MOG-antibody validation experiments organized by Euroimmun (Germany). K. Rostásy reports no disclosures relevant to the manuscript. Go to Neurology.org for full disclosures.

Received December 1, 2016. Accepted in final form June 6, 2017.

\section{REFERENCES}

1. Baumann M, Sahin K, Lechner C, et al. Clinical and neuroradiological differences of paediatric acute disseminating encephalomyelitis with and without antibodies to the myelin oligodendrocyte glycoprotein. J Neurol Neurosurg Psychiatry 2015;86:265-272.

2. Di Pauli F, Mader S, Rostasy K, et al. Temporal dynamics of anti-MOG antibodies in CNS demyelinating diseases. Clin Immunol 2011;138:247-254. 
3. Pröbstel AK, Dornmair K, Bittner R, et al. Antibodies to MOG are transient in childhood acute disseminated encephalomyelitis. Neurology 2011;77:580-588.

4. Mayer MC, Breithaupt C, Reindl M, et al. Distinction and temporal stability of conformational epitopes on myelin oligodendrocyte glycoprotein recognized by patients with different inflammatory central nervous system diseases. J Immunol 2013;191:3594-3604.

5. Rostasy K, Mader S, Schanda K, et al. Anti-myelin oligodendrocyte glycoprotein antibodies in pediatric patients with optic neuritis. Arch Neurol 2012;69: 752-756.

6. Huppke P, Rostasy K, Karenfort M, et al. Acute disseminated encephalomyelitis followed by recurrent or monophasic optic neuritis in pediatric patients. Mult Scler 2013; 19:941-946.

7. Rostasy K, Mader S, Hennes EM, et al. Persisting myelin oligodendrocyte glycoprotein antibodies in aquaporin-4 antibody negative pediatric neuromyelitis optica. Mult Scler 2013;19:1052-1059.

8. Mader S, Gredler V, Schanda, et al. Complement activating antibodies to myelin oligodendrocyte glycoprotein in neuromyelitis optica and related disorders. J Neuroinflammation 2011;8:184

9. Höftberger R, Sepulveda M, Armanque T, et al. Antibodies to MOG and AQP4 in adults with neuromyelitis optica and suspected limited forms of the disease. Mult Scler 2015;21:866-874.

10. Baumann M, Hennes E, Schanda K, et al. Children with multiphasic disseminated encephalomyelitis and antibodies to the myelin oligodendrocyte glycoprotein (MOG): extending the spectrum of MOG antibody positive diseases. Mult Scler 2016;22:1821-1829.

11. Ketelslegers IA, Van Pelt DE, Bryde S, et al. Anti-MOG antibodies plead against MS diagnosis in an Acquired Demyelinating Syndromes cohort. Mult Scler 2015;21: 1513-2030.

12. Hacohen Y, Absoud M, Deiva K, et al. Myelin oligodendrocyte glycoprotein antibodies are associated with a non-MS course in children. Neurol Neuroimmunol Neuroinflamm 2015;2:e81.

13. Krupp LB, Tardieu M, Amato MP, et al. International Pediatric Multiple Sclerosis Study Group criteria for pediatric multiple sclerosis and immune-mediated central nervous system demyelinating disorders: revisions to the 2007 definitions. Mult Scler 2013;19:1261-1267.

14. Polman $\mathrm{CH}$, Reingold SC, Banwell B, et al. Diagnostic criteria for multiple sclerosis: 2010 revisions to the McDonald criteria. Ann Neurol 2011;69:292-302.

15. Wingerchuk DM, Banwell B, Bennett JL, et al. International consensus diagnostic criteria for neuromyelitis optica spectrum disorders. Neurology 2015;85:177-189.

16. Lechner C, Baumann M, Hennes EM, et al. Antibodies to MOG and AQP4 in children with neuromyelitis optica and limited forms of the disease. J Neurol Neurosurg Psychiatry 2016;87:897-905.
17. Fernandez-Carbonell C, Vargas-Lowy D, Musallam A, et al. Clinical and MRI phenotype of children with MOG antibodies. Mult Scler 2016;22:174-184.

18. McLaughlin KA, Chitnis T, Newcombe J, et al. Agedependent B cell autoimmunity to a myelin surface antigen in pediatric multiple sclerosis. J Immunol 2009;183: 4067-4076.

19. Jarius S, Ruprecht K, Kleiter I, et al. MOG-IgG in NMO and related disorders: a multicenter study of 50 patients. Part 2: epidemiology, clinical presentation, radiological and laboratory features, treatment responses, and longterm outcome. J Neuroinflammation 2016;13:280.

20. Sepúlveda M, Armanque T, Martinez-Hernandez E, et al. Clinical spectrum associated with MOG autoimmunity in adults: significance of sharing rodent MOG epitopes. J Neurol 2016;263:1349-1360.

21. Waters P, Woodhall M, O'Connor KC, et al. MOG cellbased assay detects non-MS patients with inflammatory neurologic disease. Neurol Neuroimmunol Neuroinflamm 2015;2:e89.

22. Spadaro M, Gerdes LA, Krumbholz M, et al. Autoantibodies to MOG in a distinct subgroup of adult multiple sclerosis. Neurol Neuroimmunol Neuroinflamm 2016;3: e257.

23. Spadaro M, Gerdes LA, Mayer MC, et al. Histopathology and clinical course of MOG-antibody-associated encephalomyelitis. Ann Clin Transl Neurol 2015;2:295-301.

24. Di Pauli F, Höftberger R, Reindl M, et al. Fulminant demyelinating encephalomyelitis: Insights from antibody studies and neuropathology. Neurol Neuroimmunol Neuroinflamm 2015;2:e175.

25. Jarius S, Metz I, König FB, et al. Screening for MOG$\mathrm{IgG}$ and 27 other anti-glial and anti-neuronal autoantibodies in "pattern II multiple sclerosis" and brain biopsy findings in a MOG-IgG-positive case. Mult Scler 2016;22: 1541-1549.

26. Ramanathan S, Dale RC, Brilot F. Anti-MOG antibody: the history, clinical phenotype, and pathogenicity of a serum biomarker for demyelination. Autoimmun Rev 2016;15:307-324.

27. Jarius S, Ruprecht K, Kleiter I, et al. MOG-IgG in NMO and related disorders: a multicenter study of 50 patients. Part 1: frequency, syndrome specificity, influence of disease activity, long-term course, association with AQP4IgG, and origin. J Neuroinflammation 2016;13:279.

28. Ramanathan S, Reddel SW, Henderson A, et al. Antibodies to myelin oligodendrocyte glycoprotein in bilateral and recurrent optic neuritis. Neurol Neuroimmunol Neuroinflamm 2014;1:e40.

29. Sato DK, Callegaro D, Lana-Peixoto MA, et al. Distinction between MOG antibody-positive and AQP4 antibody-positive NMO spectrum disorders. Neurology 2014;82:474-481.

30. Ramanathan S, Prelog K, Barnes EH, et al. Radiological differentiation of optic neuritis with myelin oligodendrocyte glycoprotein antibodies, aquaporin- 4 antibodies, and multiple sclerosis. Mult Scler 2016;22:470-482. 


\section{Neurology}

\section{Prognostic relevance of MOG antibodies in children with an acquired demyelinating syndrome}

Eva-Maria Hennes, Matthias Baumann, Kathrin Schanda, et al. Neurology 2017;89;900-908 Published Online before print August 2, 2017

DOI 10.1212/WNL.0000000000004312

This information is current as of August 2, 2017

\section{Updated Information \& Services}

Supplementary Material

\section{References}

\section{Subspecialty Collections}

Permissions \& Licensing

\section{Reprints}

including high resolution figures, can be found at: http://n.neurology.org/content/89/9/900.full.html

Supplementary material can be found at: http://n.neurology.org/content/suppl/2017/08/02/WNL.0000000000004 312.DC1

http://n.neurology.org/content/supp1/2017/08/02/WNL.0000000000004 312.DC2

http://n.neurology.org/content/suppl/2017/08/28/WNL.0000000000004 312.DC3

This article cites 30 articles, 6 of which you can access for free at: http://n.neurology.org/content/89/9/900.full.html\#\#ref-list-1

This article, along with others on similar topics, appears in the following collection(s):

\section{All Pediatric}

http://n.neurology.org//cgi/collection/all_pediatric

Information about reproducing this article in parts (figures,tables) or in its entirety can be found online at:

http://n.neurology.org/misc/about.xhtml\#permissions

Information about ordering reprints can be found online:

http://n.neurology.org/misc/addir.xhtml\#reprintsus

Neurology ${ }^{\circledR}$ is the official journal of the American Academy of Neurology. Published continuously since 1951, it is now a weekly with 48 issues per year. Copyright () 2017 American Academy of Neurology. All rights reserved. Print ISSN: 0028-3878. Online ISSN: 1526-632X.

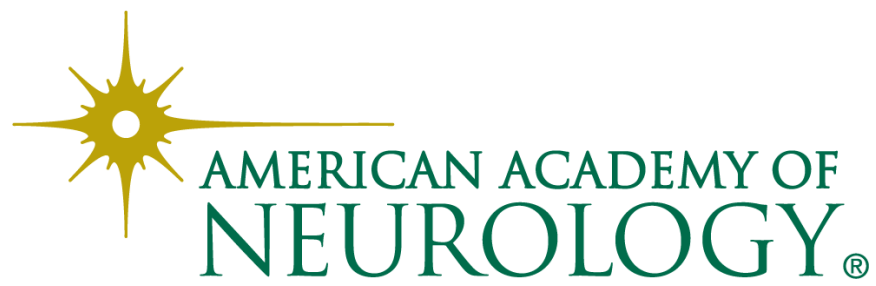

